\title{
Map-based Access to Multiple Educational On-Line Resources from Mobile Wireless Devices
}

\author{
P. Brusilovsky ${ }^{1}$ and R.Rizzo ${ }^{2}$ \\ ${ }^{1}$ School of Information Sciences, University of Pittsburgh, Pittsburgh PA 15260, USA \\ peterbasis.pitt.edu \\ ${ }^{2}$ Institute for Educational and Training Technologies, Italian National Research Council, \\ Palermo, Italy \\ rizzo@itdf.pa.cnr.it
}

\begin{abstract}
While large volumes of relevant educational resources are available currently online, almost all existing resources have been designed for relatively large screens and relatively high bandwidth. Searching for the proper interface to access multiple resources from a mobile computer we have selected an approach based on self-organized hypertext maps. This paper presents our approach and its implementation in the KnowledgeSea system. It also discusses the ongoing work on using our approach with very narrow screens of Palm-like devices.
\end{abstract}

\section{Introduction}

Large volumes of relevant educational resources are available currently on the Web for the students. Altogether, they well complement course textbooks and enable the students with different level of knowledge or different learning styles to get a better comprehension of the subject. The current model of accessing these resources from computers at home or at the university labs is a restriction - like a requirement to read a textbook always at home or in class, but not outside, in a café, or while riding a bus. The use of mobile wireless handheld devices potentially allows the students to access educational resources really "anywhere". Moreover, the success of mobile e-books shows that the users are quite willing to read predominantly textual sources on mobile devices. The bottleneck here is finding a relevant educational resource that currently demands large screens and high bandwidth (Figure 1).

Searching for the proper interface to access multiple resources on a mobile computer we have considered several options and finally selected an approach based on self-organized hypertext maps. This paper presents our approach and its current implementation - the KnowledgeSea system that can be used successfully with a number of mobile devices with landscape-style screen (such as HP Jornada organizers). It also discusses the ongoing work on using our approach with more narrow screens of Palm-like devices. 


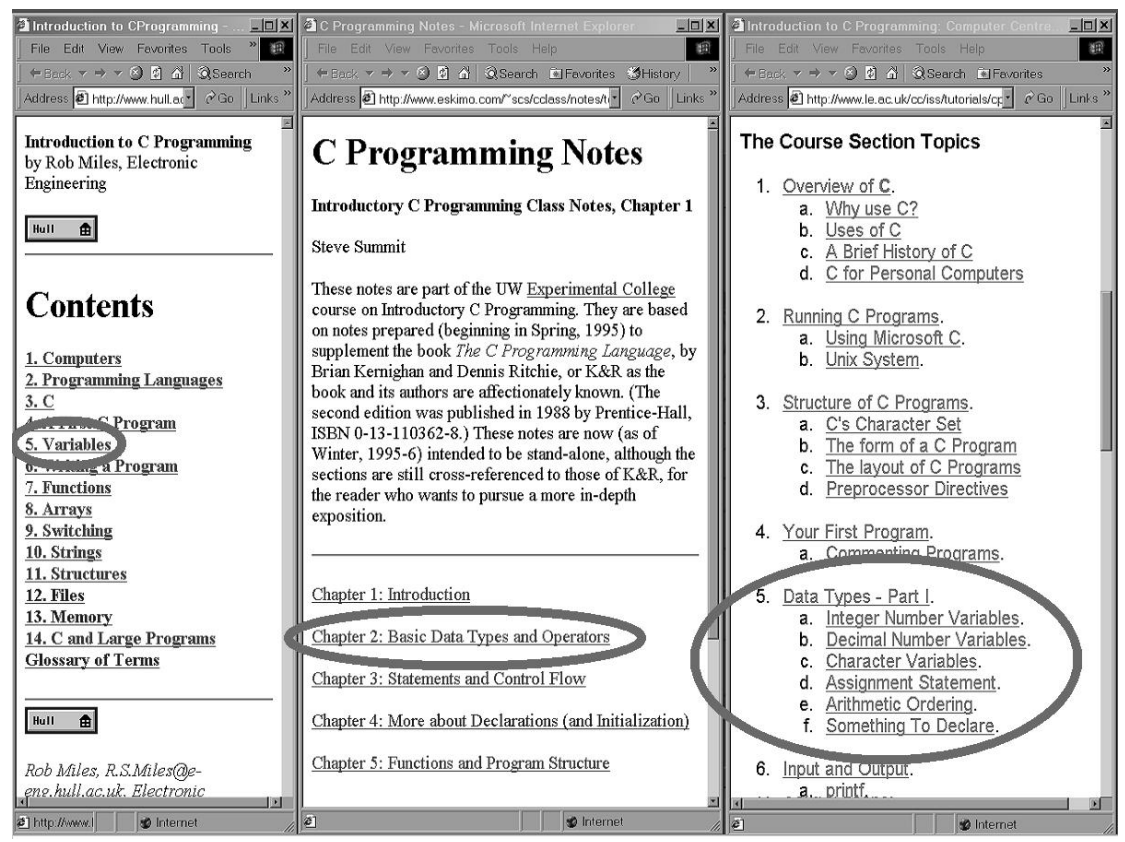

Fig. 1. Selecting relevant reading sections in multiple educational resources is a challenging task that demands good interface skills, large screen and fast Internet connection.

\section{The KnowledgeSea Approach}

The core of our approach to navigating educational resources is a self-organized hyperspace map automatically built using a Self-Organizing neural Map (SOM) [2]. SOM is a very attractive technology for developing compact maps of large hyperspaces [3] since it builds a map representing the neighborhood relationship between the objects. A two-dimensional 8-by-8 map of educational resources developed with SOM technology is a core of our KnowledgeSea system for mapbased access to multiple educational resources (Figure 2). KnowledgeSea was designed to support a typical university class on $\mathrm{C}$ programming. In this context, the goal of the students is to find most helpful Web-based material as a part of readings assigned for every lecture in the course. The most easily available Web educational resources are multiple hypertextual $\mathrm{C}$ tutorials (see for example http://www.le.ac.uk/cc/iss/tutorials/cprog/cccc.html). In this context, the goal of KnowledgeSea system to help the user to navigate from lectures to relevant tutorial pages in multiple tutorials and between them. The main component of the interface is a KnowledgeSea map - a table in which each cell is used to group together a set of educational resources (Figure 2). The map is organized in a way that resources (web pages) that are semantically related are close to each other on the map. Resources 
located in the same cell are considered very similar; resources located in directly connected cells are reasonably similar and so on.

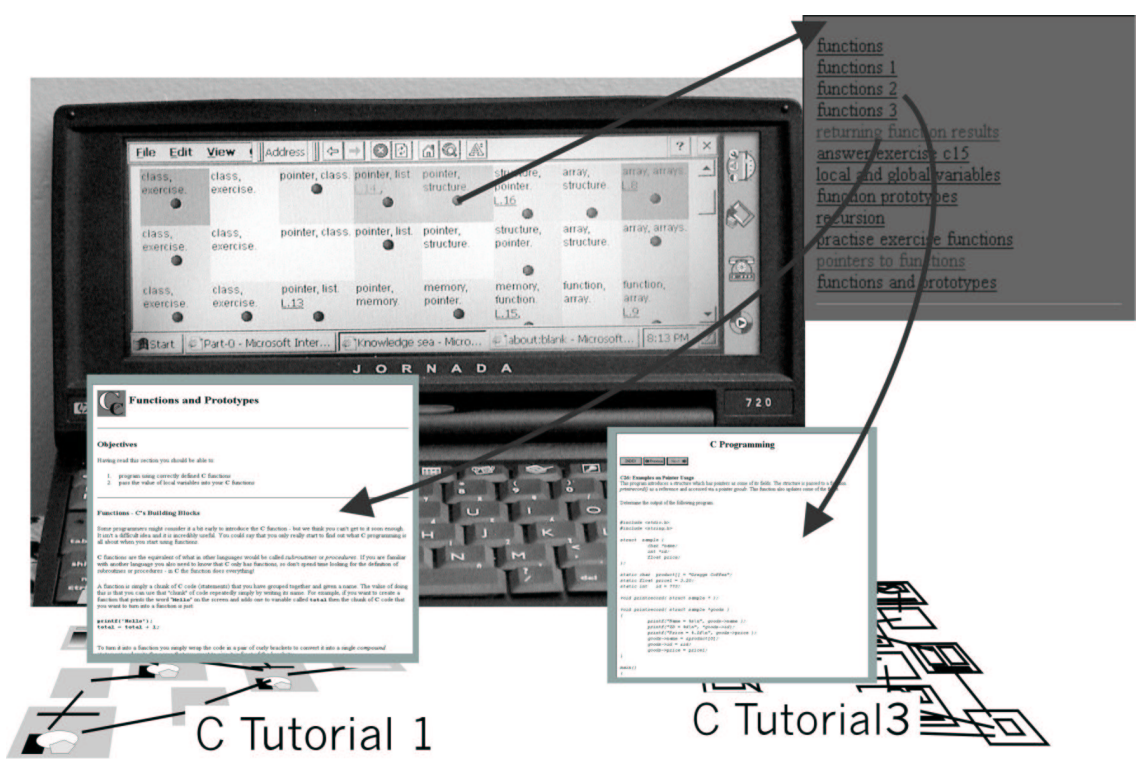

Fig. 2. A session of work with the KnowledgeSea system.

Each cell displays a set of keywords that helps the user to locate the relevant section on the map. Some cells also display links to "critical" resources that serve as origin points for horizontal navigation. The map serves as a mediator to help the user navigate from critical resources to related resources. For lecture-to-tutorial navigation the critical resources are lectures and lecture slides. The presence of related educational resources in the cell is indicated by a red dot. The cell color indicates the "depth of the information sea" - the number of resource pages lying "under" the cell. A click on the red dot opens a cell content window (right on Figure 2) that provides a list of links to all tutorial pages relevant to this cell. A click on any of these links will open a resource-browsing window with the selected relevant page from one of the tutorial. This page is loaded "as is" from its original URL.

The functionality and the usefulness of our map-based information access approach was evaluated it in the context of a real Programming and Data Structures course at the University of Pittsburgh. The characteristics of the SOM, the implementation details of the KnowledgeSea system, and some results of this study were reported in [1]. We were very encouraged to find that about $2 / 3$ of the 21 students participated in the study thought that the system has achieved the goal of providing an access to the online C tutorials completely or "quite well". The overall idea to attach the resources to cells on a map and show where the lecture belongs was considered "very easy" by the $19 \%$ of the students and "quite easy" by the $42.8 \%$. Interesting is that when asked in which context they would expect to use the KnowledgeSea system from a mobile device, the majority of the students have selected only home or library. Only few of 
them have indicated an interest to use the system in a bus, park or from "anywhere when I have some spare time". It is not clear yet whether this answer was caused by the nature of information access task, the nature of the system or simply by the students' prejudice to using computers in a familiar context.

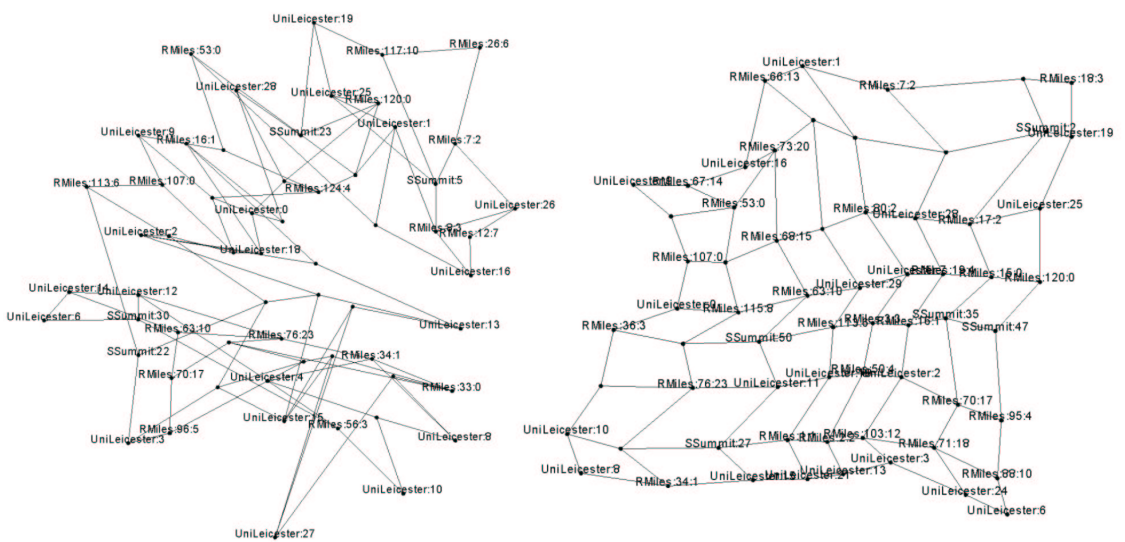

Fig. 3. A representation of the two different map geometries after the learning phase. On the left the $4 \times 15$ map and on the right the $8 \times 8$ map. The cells are labeled using a reference to the Web pages of the tutorial.

\section{Current and Future Work: The Narrow Screen Challenge}

The original platform for our experiments was a desktop PC and the HP Jornada organizer with a relatively wide screen. In this context we have been working with an 8x8 SOM map (Figure 2). The "learning" (organizing) stage of the SOM map in this case was not complicated and the standard value of parameter sufficient. Overall, we can conclude that SOM-based access to multiple information resources in the investigated context is a very useful technology. The $8 \times 8$ map that we have explored has worked well for the students. This map is large enough to provide a reasonable split of diverse content, yet is small enough to fit a Jornada-like handheld. We plan to continue investigating the same map and the same interface for the context of a larger hyperspace of educational material (6 or more external tutorials instead of 3 ).

Our new challenge is to make our technology work on a narrow Palm-like screen that is more typical for handheld organizers. As soon as wireless cards become available for the Palm platform, we have started to experiment with Palm devices equipped with BlaserTM Web browser. We have found that with this browser the narrow Palm screen can fit only 3-4 cells of our original text-based map in a row. Since we want to avoid horizontal scrolling, we have started with exploring an unusual 4x15 map geometry. Unfortunately, for this geometry the learning stage of the SOM map was more complicated and the results are not satisfying even with extra efforts to split the learning phase in three sessions and to use non-standard values of 
the parameters. The four-cell width was apparently not enough to organize an information space without "twisting" as it can be seen on Fig. 3. In this figure it is possible to see the lattice of the network obtained after the learning stage. The lattice is irregular and this is the reason why the resulting map did not look intuitive and contained too many cells with no information items. We concluded that this map could be more confusing than helpful for the students.

Instead of continuing our experiments with narrow maps we are going to stay with wider maps and now attempting to develop a better interface for using reliable $8 \times 8$ maps on Palm platform. Our intention is to change the visualization metaphor to make the system suitable also for the smaller Palm-style wireless devices. At the moment we are considering two options: to switch from text-based visualization to graphical metaphor to fit the PDA screen or to use hierarchical maps. Although hierarchies can be difficult to manage in this application they can be a feasible way to guide the user in navigation.

\section{References}

[1] Brusilovsky, P. and Rizzo, R.: Map-Based Horizontal Navigation in Educational Hypertext. In: Proc. of 13th ACM Conference on Hypertext and Hypermedia (Hypertext 2002), College Park, MD, ACM (2002) in press

[2] Kohonen, T.: Self-Organizing Maps. Springer Verlag, Berlin

[3] Kohonen, T., Kaski, S., Lagus, K., and Honkela, T.: Very Large Two-Level SOM for the Browsing of the Newsgroups. In: Proc. of ICANN'96, Berlin, Springer Verlag (1996) 269274 